\title{
Erratum: Spatial optical solitons in highly nonlocal media [Phys. Rev. A 91, 013841 (2015)]
}

\author{
Alessandro Alberucci, Chandroth P. Jisha, Noel F. Smyth, and Gaetano Assanto \\ (Received 28 February 2017; published 25 May 2017)
}

DOI: 10.1103/PhysRevA.95.059910

The purpose of this Erratum is to amend some of the power values reported in our paper. In fact, we mistakenly adopted two distinct expressions for the same physical quantity in the above-named paper and in our previous paper, Ref. [1]. The different expressions used for the index well, written as $\Delta n^{2}$ in Ref. [1] and as $2 n_{0} \Delta n$ in our paper, result in an incorrect estimation of power by a factor 2 . As a direct consequence, Eq. (17) in our paper should read

$$
w_{s}(P)=\sqrt{\frac{8 \pi}{n_{0} k_{0}^{2} \alpha P}},
$$

as kindly pinpointed in Ref. [2]. Due to the incorrect expression, Fig. 7 and the corresponding caption in our paper need to be replaced by Fig. 1. The right inline equation before Eq. (17) is then

$$
\alpha n_{0} P w^{2} k_{0}^{2}=4 \pi \text {. }
$$

The correct form of the breathing period $\Lambda$ and the coefficient $\phi_{2}$, appearing in the text after Eq. (19), are

$$
\begin{aligned}
\Lambda & =\frac{4 \sqrt{2} \pi^{2}}{\alpha k_{0} P}, \\
\phi_{2} & =-\frac{n_{0} k_{0}^{2} \alpha^{2} P^{2}}{16 \pi^{2}} .
\end{aligned}
$$

The other figures, expressions, and results in our paper are not affected and remain valid.
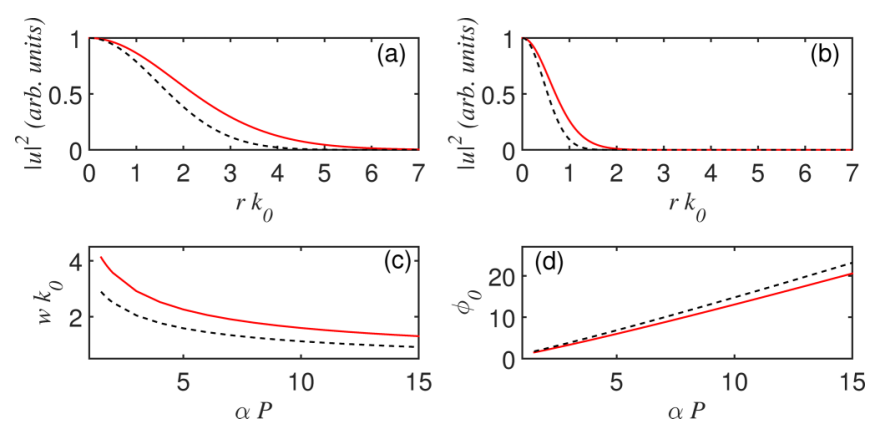

FIG. 1. Soliton profile $|u|^{2}$ normalized to its own peak vs normalized radial distance $r k_{0}$ for (a) $\alpha P=1.5$ and (b) $\alpha P=15$, respectively; (c) normalized soliton width $w k_{0}$; and (d) peak of the nonlinear perturbation $\phi_{0}$ vs normalized power $\alpha P$. In all panels, red solid lines and black dashes correspond to exact numerical solutions and predictions of the original Snyder-Mitchell model, respectively (see Ref. [1] for deeper insight).

[1] A. Alberucci, C. P. Jisha, and G. Assanto, Accessible solitons in diffusive media, Opt. Lett. 39, 4317 (2014).

[2] M. S. Petrović, N. B. Aleksić, B. N. Aleksić, A. I. Strinić, and M. R. Belić, Comment on "Spatial optical solitons in highly nonlocal media", Phys. Rev. A 95, 057801 (2017). 